\title{
Bacterial additives improve the water resistance of mortar
}

\author{
Marvin Johannes Ertelt ${ }^{1,2}$, Manuel Raith ${ }^{2}$, Josef Eisinger ${ }^{1}$, Christian U. Grosse ${ }^{2}$ \\ and Oliver Lieleg ${ }^{1, \#}$ \\ 1 Department of Mechanical Engineering and Munich School of Bioengineering, \\ Technical University of Munich, Boltzmannstrasse 11, 85748 Garching, Germany, \\ e-mail: oliver.lieleg@tum.de \\ 2 Center for Building Materials, Chair of Non-destructive Testing, \\ Technical University of Munich, Franz-Langinger-Strasse 10, 81245 Munich, Germany, \\ e-mail: grosse@tum.de \\ \#: correspondence: oliver.lieleg@tum.de
}

Total number of pages: 6

Total number of figures: 8

Total number of tables: 0 


\section{Detailed manufacturing process of the different bacterial additives}

\subsection{Overnight culture:}

$10 \mathrm{~mL}$ of liquid Luria/Miller LB-Medium (10 g/L tryptone, $5 \mathrm{~g} / \mathrm{L}$ yeast extract, $10 \mathrm{~g} / \mathrm{L}$ sodium chloride, $\mathrm{pH} 7.0 \pm 0.2$, Carl-Roth, Karlsruhe, Germany) were inoculated with a frozen bacterial/glycerol stock. After incubation at $37^{\circ} \mathrm{C}$ and $90 \mathrm{rpm}$ (or $200 \mathrm{rpm}$ for $B$. subtilis $\mathrm{B}-1$ ) in a shaking incubator (Sartorius, Göttingen, Germany) overnight, a solution of planktonic bacteria (= an 'overnight culture') was obtained (Figure S1).
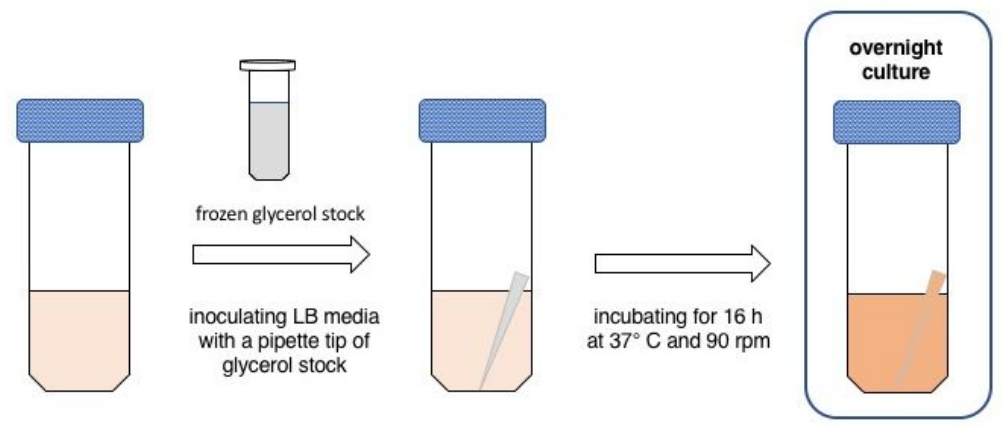

Figure S1: Preparation of bacterial overnight cultures. Each overnight culture was incubated for $16 \mathrm{~h}$ to reach the stationary phase of the bacteria growth rate and thus ensure the highest possible density of live planktonic bacteria in the liquid media.

\subsection{Bacterial biofilm:}

To generate bacterial biofilm, agar plates were produced first. Therefore, $20 \mathrm{~g}$ LB media and $12 \mathrm{~g}$ Agar were dissolved in $800 \mathrm{~mL}$ distilled water, and subsequently the solution was autoclaved. The mixture (hereafter referred to as LB-agar) was allowed to partially cool down to $60^{\circ} \mathrm{C} .25 \mathrm{~mL}$ of (still liquid) LB-agar was then poured into a sterile petri dish and allowed to fully cool down. After the LB-agar had solidified, $100 \mu \mathrm{L}$ overnight culture was plated on every agar-filled Petri dish and incubated at $37^{\circ} \mathrm{C}$. After $24 \mathrm{~h}$, a continuous layer of biofilm has formed on the agar plates (Figure S2).

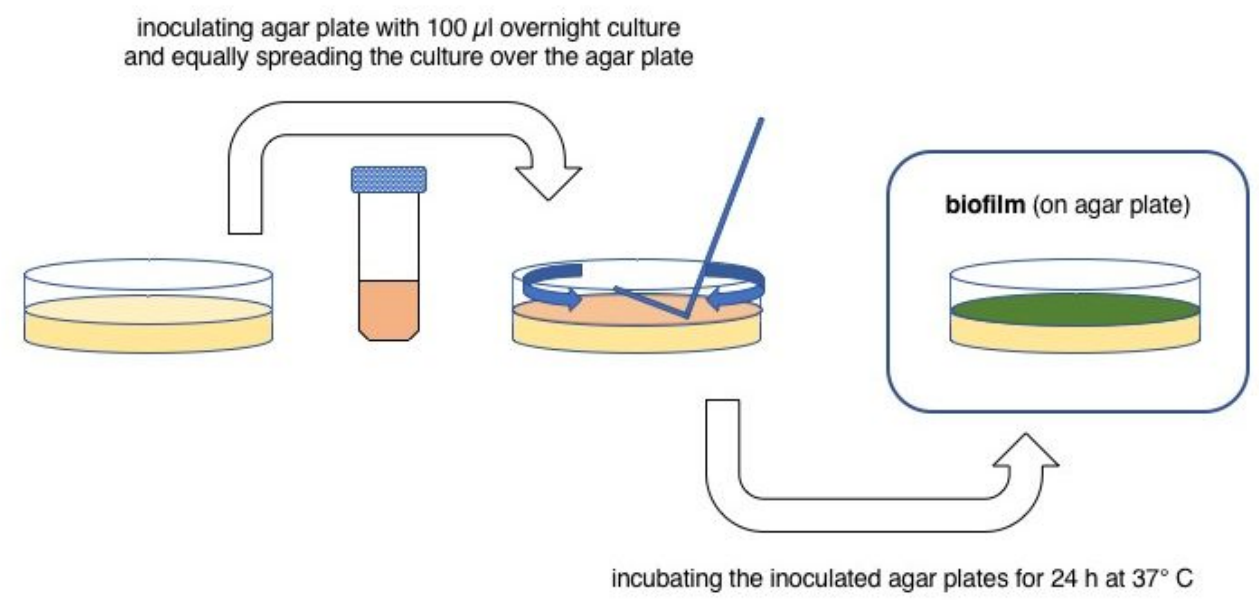

Figure S2: Formation of bacterial biofilm on agar plates. $100 \mu \mathrm{L}$ of Overnight culture (highly concentrated solution of planktonic bacteria) was applied on and evenly distributed over each agar plate. On the nutrient rich agar, the bacteria start to produce bacterial biofilm. 
Finally, using a customized PDMS-spatula, the generated biofilm could be harvested without collecting any agar pieces (Figure S3).

a

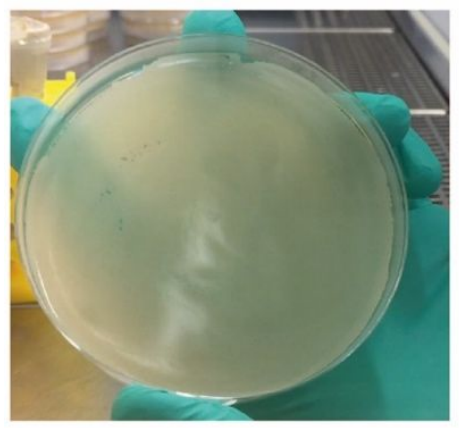

d

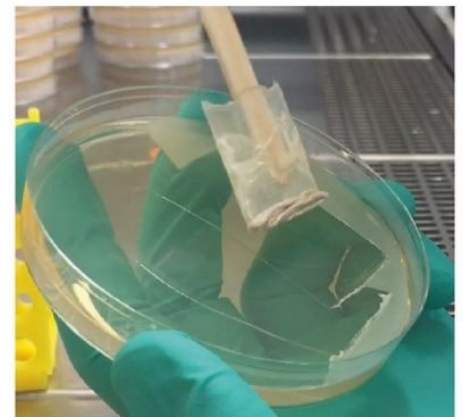

b

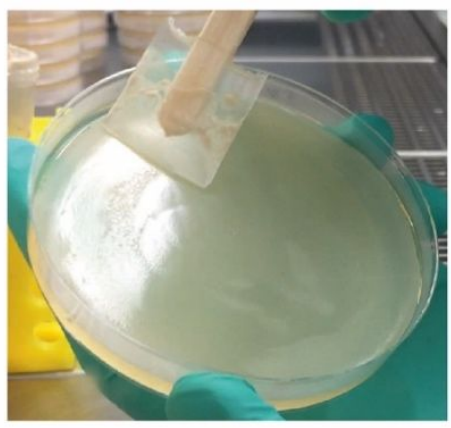

e

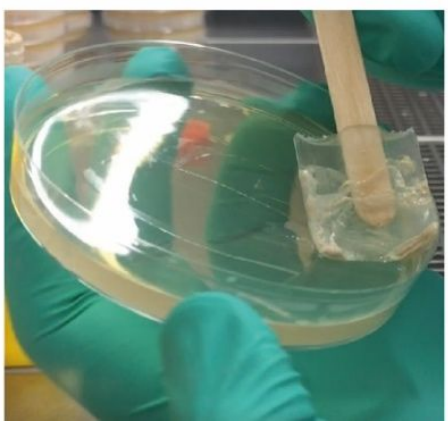

C

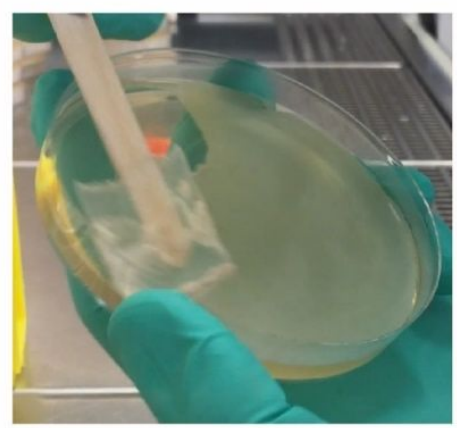

$f$

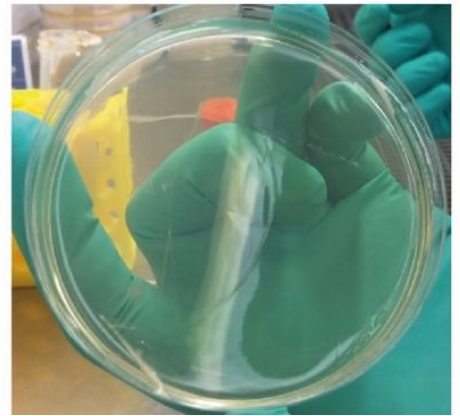

Figure S3: Harvesting process to collect bacterial biofilm from agar plates using a PDMS spatula. a) After incubating the inoculated agar plates for 24 hours, a continuous biofilm layer is formed on the whole agar surface. b) Using a custom-made PDMS-spatula, the biofilm can be collected from the agar (c - e) without damaging the agar substrate $(\mathrm{f})$.

\subsection{Biofilm powder:}

Bacterial biofilm was generated and collected as described above, and then stored at $-80^{\circ} \mathrm{C}$ for at least $2 \mathrm{~h}$. Subsequently, the frozen biofilm was freeze-dried for $72 \mathrm{~h}$. To obtain biofilm powder, the freeze-dried biofilm was finely ground using a mortar and pestle.

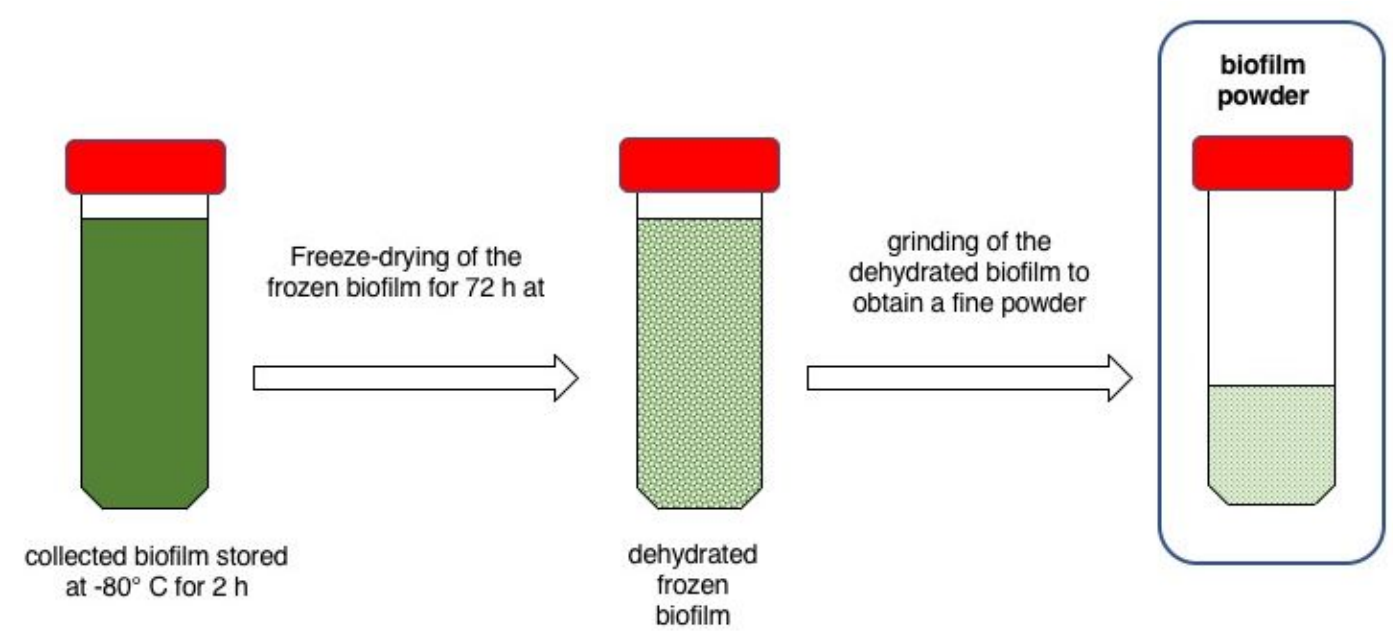

Figure S4: Production procedure to obtain a powder from a freshly collect, wet bacterial biofilm. After collection, the biofilm was dehydrated in a freeze-drying process, and finally the dehydrated biofilm was finely ground into a powder. 


\section{Optical characterization of the microbial composition of the different bacterial additives}

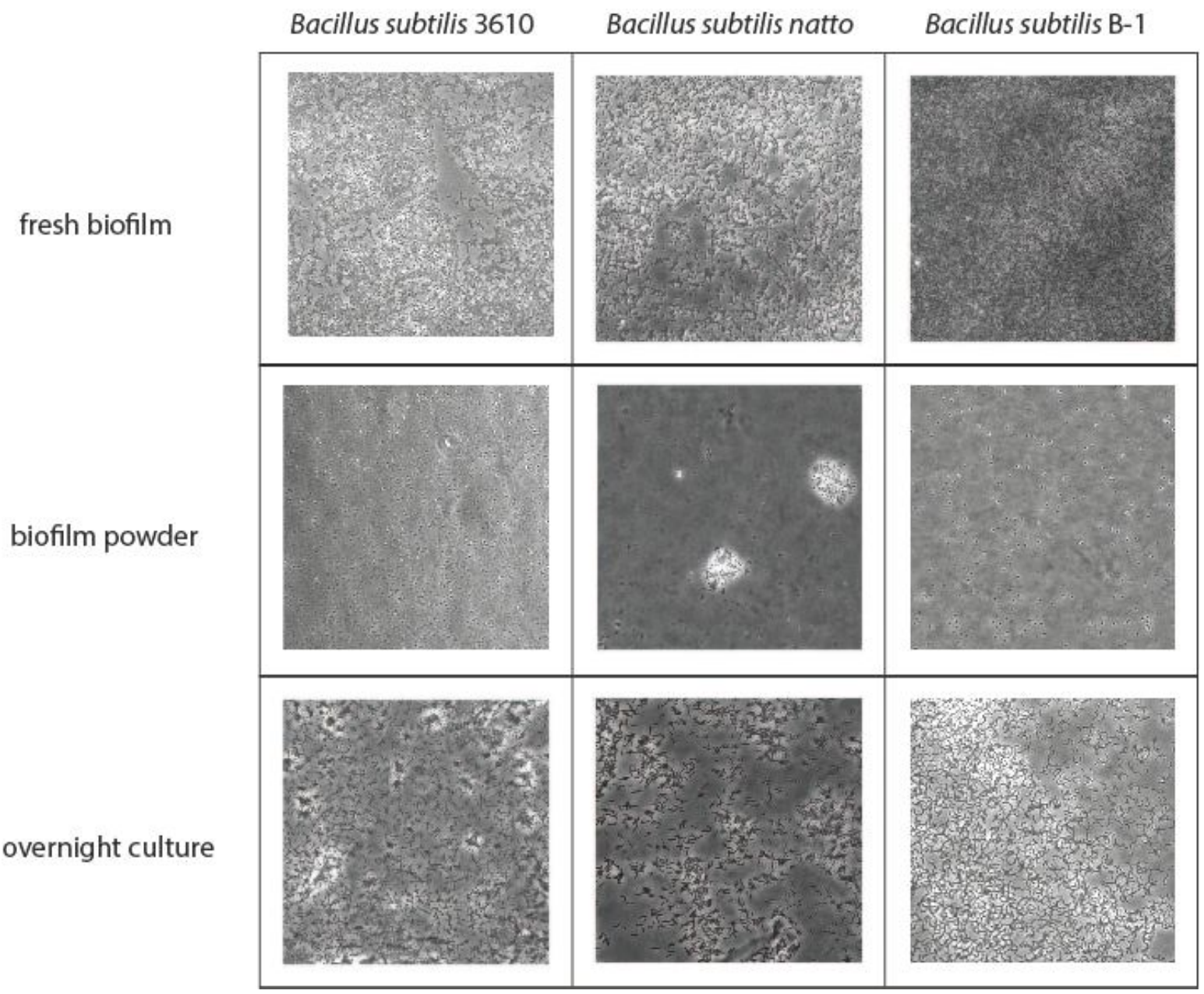

Figure S5: Phase contrast images of the nine different bacterial additives used in this study. Fresh biofilm (top row) contains a mixture of active cells (rod shaped objects) and spores (circular objects), i.e., bacteria in a metabolic inactive state. In contrast, biofilm powder (middle row) contains exclusively spores, and bacterial suspensions ('overnight culture') contain mostly active bacteria cells and almost no spores. 


\section{Influence of bacterial growth media (components) on the material properties of mortar}

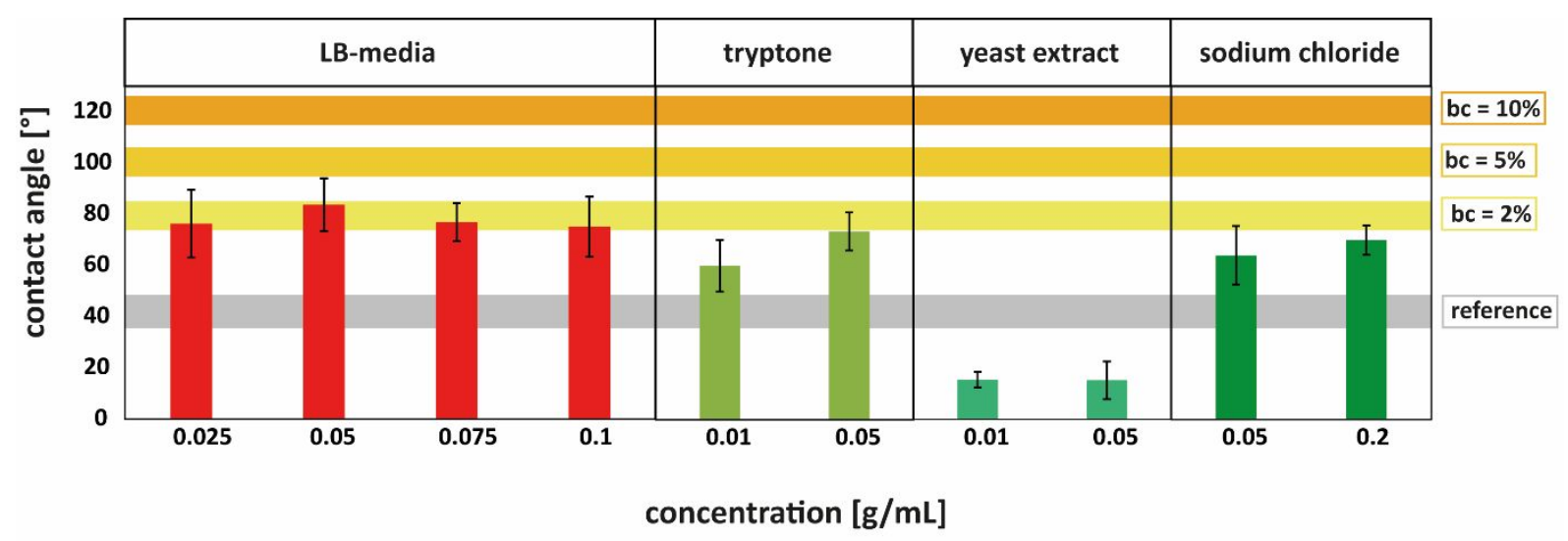

Figure S6: Contact angles obtained for mortar samples containing different concentrations of LB-media and single media components, respectively. Standard LB-media $(0.025 \mathrm{~g} / \mathrm{mL})$ used for the cultivation of bacteria as described in the main paper contains tryptone $(0.010 \mathrm{~g} / \mathrm{mL})$, yeast extract $(0.005 \mathrm{~g} / \mathrm{mL})$ and sodium chloride $(0.010 \mathrm{~g} / \mathrm{mL})$. If those components are added to mortar alone (i.e., without biofilm components or bacteria), the measured contact angles never reach the values above $85^{\circ}$ and thus are much lower than what we obtain with the different bacterial additives. The contact angle values obtained for biofilm additives (as described in the main text) are indicated by the three yellow/orange stripes and refer to samples with different biofilm content.

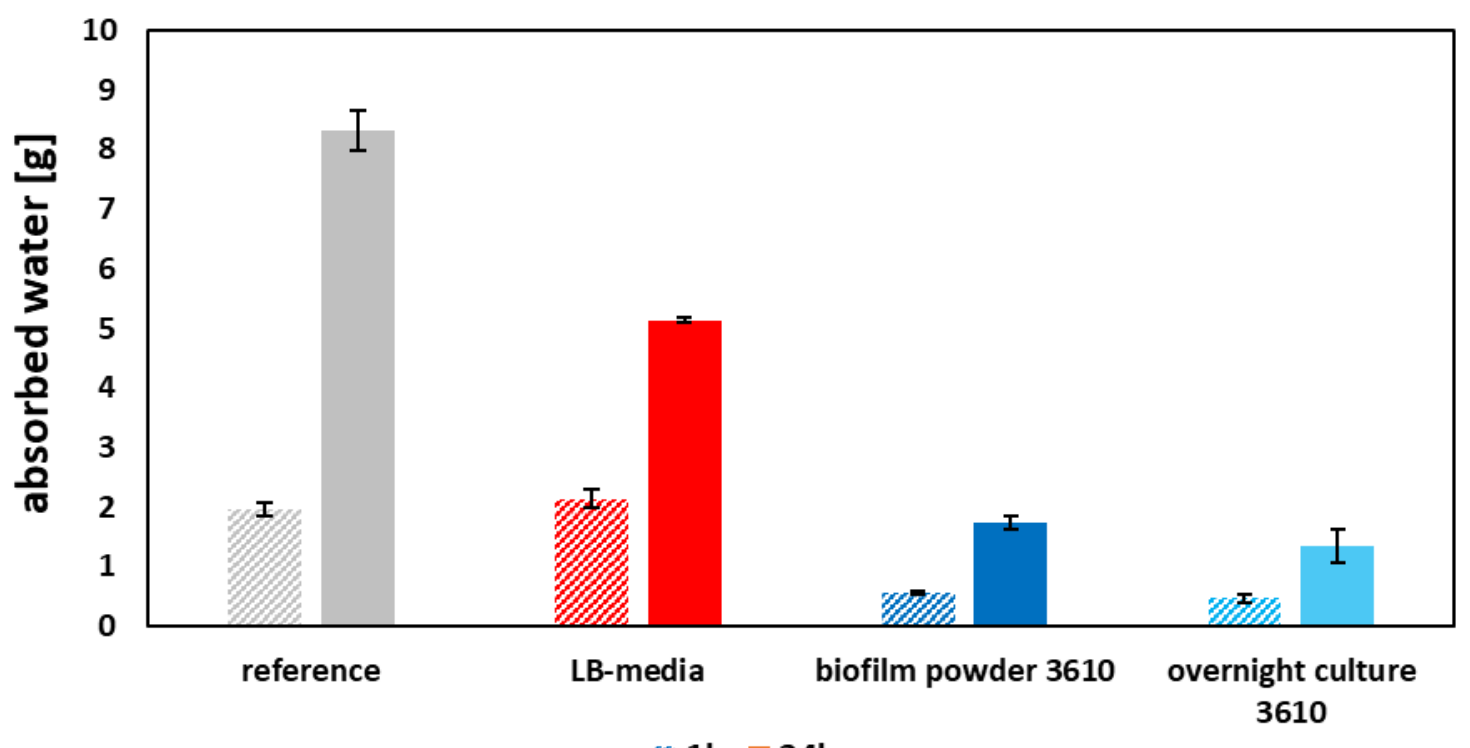

Figure S7: Capillary water uptake into hybrid mortar samples. The amount of water absorbed by the different samples after partial immersion into a water bath is depicted for immersion times of $1 \mathrm{~h}$ and $24 \mathrm{~h}$, respectively. Samples enriched with biofilm powder ( $b c=2 \%$, left) and samples enriched with a bacterial overnight culture $(O D>1.0$, right) are compared to both, unmodified control samples (grey bars) and samples containing culture media. The values shown represent averages of three measurements conducted on independent samples, error bars denote the standard deviation. 


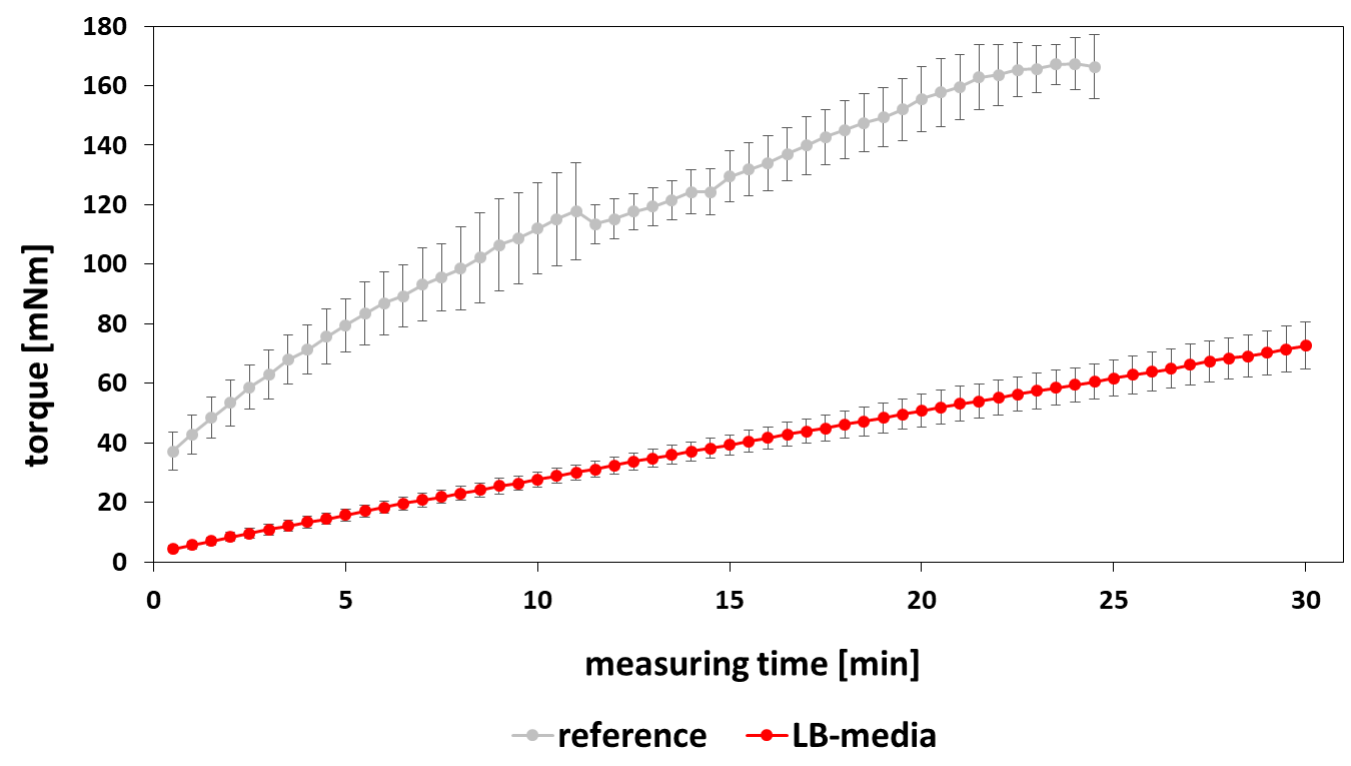

Figure S8: Influence of LB media on the hardening process of mortar. The graphs depict the time-dependent increase of the torque require to maintain a constant shear rate of $0.001 \mathrm{~s}^{-1}$ on different mortar variants. Mortar samples containing LB media instead of mixing water (red curve) are compared to unmodified reference samples (grey curve). The values shown represent averages of three independent measurements, error bars denote the standard deviation. 